\title{
Molecule 5
}

\section{Singlet}

\section{UHF/STO-3G}

1 | 1 | UNPC-UNK |FOpt | UHF |STO-3G |C12H10 |PCUSER | 19-Oct-2002|0|| \#UHF STO-3G OPT GUESS $=($ ALWAYS, MIX) || molecule 38 || $0,1 \mid \mathrm{C}, 1.4356102764,-0.0002978046$, $0.5791783984|\mathrm{C}, 1.4183551147,-0.0004440101,1.9424280111| \mathrm{H}, 2.3595988335$, $-0.0006533492,2.4767798248|\mathrm{C}, 0.218288979,-0.0003322494,2.6936104445| \mathrm{H}$, $0.2652728457,-0.0004561469,3.7750432207 \mid C,-0.9657833426,-0.0000680555$, $2.0447606528|\mathrm{H},-1.9020256622,0.0000275258,2.5872519397| \mathrm{C},-1.009971698$, $0.0000938371,0.617632139|\mathrm{C},-2.289252595,0.0003747308,-0.026516816| \mathrm{H},-3$ $.173368388,0.0004574103,0.5991043315 \mid C,-2.3938704384,0.0005541691,-1.3$ $723160677|\mathrm{H},-3.3587261306,0.0007815211,-1.8619550394| \mathrm{C},-1.2108851303,0$ $.0004441715,-2.1529755588|\mathrm{H},-1.3077923279,0.0006014726,-3.2320910714| \mathrm{C}$ $, 0.0410592842,0.0001506803,-1.5981773861 \mid C, 1.1972653903,0.0000939782$,$2.5371664515|\mathrm{H}, 0.878929188,0.0001642272,-3.5907264965| \mathrm{C}, 0.1811381954,-$ $0.0000195562,-0.1464315624|\mathrm{C}, 2.8264645647,-0.0004547585,-0.065002395| \mathrm{H}$ $, 2.7853204137,-0.0003074538,-1.1441077178 \mid \mathrm{H}, 3.380988309,-0.8777506487$, $0.2581657131|\mathrm{H}, 3.3812913164,0.8765646456,0.258394845| \mid$ Version=x86-Win3 $2-G 98 R e v A .11 .4|\mathrm{HF}=-454.5382336| \mathrm{S} 2=0.793303|\mathrm{~S} 2-1=0.| \mathrm{S} 2 \mathrm{~A}=0.017836 \mid \mathrm{RMSD}=8$ $.491 \mathrm{e}-009|\mathrm{RMSF}=4.529 \mathrm{e}-005| \mathrm{Dipole}=-0.3554549,0.0000624,-0.0103346 \mid \mathrm{PG}=\mathrm{C} 0$ $1[\mathrm{X}(\mathrm{C} 12 \mathrm{H} 1 \mathrm{O})]|| \mathrm{e}$

\section{$\operatorname{UCCSD}(T) / S T O-3 G$}

1 | 1 | UNPC-UNK | SP | UCCSD (T) -FC |STO-3G|C12H10 |PCUSER | 19-OCt-2002|0|| \#UCCSD -T/STO-3G GUESS=(ALWAYS, MIX) || molecule 38||0,1|C|C, 1, 1.36335882|H, 2, 1 . $08234544,1,118.85875956 \mid C, 2,1.41578028,1,122.76971795,3,179.99993287,0$ $|\mathrm{H}, 4,1.08245293,2,119.55683777,1,-179.99988531,0| \mathrm{C}, 4,1.35019754,2,119$. $23352837,1,-0.00009744,0 \mid \mathrm{H}, 6,1.0820566,4,121.1885809,2,-179.99977918,0$ $|\mathrm{C}, 6,1.42781246,4,120.49541877,2,0.00015447,0| \mathrm{C}, 8,1.43230149,6,118.499$ $85339,4,-179.99992627,0|\mathrm{H}, 9,1.08308013,8,117.98951358,6,0.00027269,0| \mathrm{C}$ $, 9,1.34985946,8,121.17140297,6,179.99918724,0 \mid \mathrm{H}, 11,1.08198562,9,121.35$ $163909,8,-179.99906764,0 \mid C, 11,1.41735087,9,118.97603238,8,0.00077548,0$ | H, 13,1.08345804,11,118.28953586, 9,-179.99932864,0|C,13,1.36936698,11, $122.67843907,9,0.00056837,0 \mid C, 15,1.48946736,13,117.01844212,11,-179.99$ $878074,0|\mathrm{H}, 16,1.10060289,15,112.26872582,13,-0.00782719,0| \mathrm{C}, 15,1.45848$ $828,13,119.41189528,11,-0.00183994,0 \mid C, 1,1.5327898,18,125.10267606,8,1$ $80 ., 0|\mathrm{H}, 19,1.07988942,1,112.66792004,18,0.00159754,0| \mathrm{H}, 19,1.08700611,1$ $, 109.75731345,18,-120.97000181,0 \mid \mathrm{H}, 19,1.08700577,1,109.75726485,18,120$ $.97325609,0||$ Version=x86-Win32-G98RevA.11.4|HF=-454.5382336|MP $2=-455.1$ $954779|\mathrm{MP} 3=-455.2854549| \mathrm{MP} 4 \mathrm{D}=-455.3213985|\mathrm{MP} 4 \mathrm{DQ}=-455.3081125| \mathrm{PUHF}=-454$ $.5246514|\mathrm{PMP} 2-0=-455.1816476| \mathrm{PMP} 3-0=-455.2713779|\mathrm{MP} 4 \mathrm{SDQ}=-455.3097196| \mathrm{C}$ $\mathrm{CSD}=-455.3441552|\mathrm{CCSD}(\mathrm{T})=-455.3750168| \mathrm{S} 2=0.793302|\mathrm{~S} 2-1=0.822626| \mathrm{S} 2 \mathrm{~A}=0$. $017836|\mathrm{RMSD}=1.994 \mathrm{e}-009| \mathrm{PG}=\mathrm{C} 01 \quad[\mathrm{X}(\mathrm{C} 12 \mathrm{H} 10)]|| @$

\section{UB3LYP/STO-3G}

1 | 1 | UNPC-UNK | SP | UB3LYP |STO-3G |C12H10 | PCUSER | 02-Feb-2003|0|| \#UB3LYP/STO 
-3G GUESS=(ALWAYS, MIX) || molecule 38||0,1|C|C, 1, 1.36335882|H, 2, 1.082345 $44,1,118.85875956|\mathrm{C}, 2,1.41578028,1,122.76971795,3,179.99993287,0| \mathrm{H}, 4,1$ $.08245293,2,119.55683777,1,-179.99988531,0 \mid C, 4,1.35019754,2,119.233528$ $37,1,-0.00009744,0|\mathrm{H}, 6,1.0820566,4,121.1885809,2,-179.99977918,0| \mathrm{C}, 6,1$ $.42781246,4,120.49541877,2,0.00015447,0 \mid C, 8,1.43230149,6,118.49985339$, $4,-179.99992627,0|\mathrm{H}, 9,1.08308013,8,117.98951358,6,0.00027269,0| \mathrm{C}, 9,1.3$ $4985946,8,121.17140297,6,179.99918724,0 \mid \mathrm{H}, 11,1.08198562,9,121.35163909$ , 8, $-179.99906764,0|\mathrm{C}, 11,1.41735087,9,118.97603238,8,0.00077548,0| \mathrm{H}, 13$, $1.08345804,11,118.28953586,9,-179.99932864,0 \mid C, 13,1.36936698,11,122.67$ $843907,9,0.00056837,0 \mid \mathrm{C}, 15,1.48946736,13,117.01844212,11,-179.99878074$ $, 0|\mathrm{H}, 16,1.10060289,15,112.26872582,13,-0.00782719,0| \mathrm{C}, 15,1.45848828,13$ $, 119.41189528,11,-0.00183994,0|C, 1,1.5327898,18,125.10267606,8,180 ., 0|$ $\mathrm{H}, 19,1.07988942,1,112.66792004,18,0.00159754,0 \mid \mathrm{H}, 19,1.08700611,1,109.7$ $5731345,18,-120.97000181,0 \mid \mathrm{H}, 19,1.08700577,1,109.75726485,18,120.97325$ 609,0||Version=x86-Win32-G98RevA.11.4|HF=-457.4928878|S2=0.753687|S2-1 $=0 .|S 2 A=0.006592| R M S D=5.738 e-005 \mid D i p o l e=-0.4535517,-0.0000291,0.119103$ $7|\mathrm{PG}=\mathrm{C} 01 \quad[\mathrm{X}(\mathrm{C} 12 \mathrm{H} 10)]| \mid$

\section{UHF/4-31G}

1 | 1 | UNPC-UNK | FOpt |UHF | 4-31G |C12H10|PCUSER | 26-Nov-2002|0|| \#UHF 4-31G OP $\mathrm{T}$ GUESS=(ALWAYS, MIX) || molecule 38||0,1|C,1.4428464513,-0.0000010176, 0. $5467995797|\mathrm{C}, 1.4343650633,-0.0000020069,1.9155205655| \mathrm{H}, 2.3762583877,-0$ $.0000078015,2.4299323247|\mathrm{C}, 0.2580895593,0.0000037945,2.6750503901| \mathrm{H}, 0$. $3145834127,0.0000030688,3.7452438414 \mid \mathrm{C},-0.9370839887,0.0000083749,2.03$ $99015119|\mathrm{H},-1.856058933,0.0000109942,2.592619821| \mathrm{C},-0.9959366522,0.000$ $0076804,0.6293654626|\mathrm{C},-2.2708466017,0.000006877,0.0108200085| \mathrm{H},-3.137$ $7154419,0.0000114074,0.6425540592 \mid \mathrm{C},-2.4033152528,-0.0000066922,-1.335$ $3838771|\mathrm{H},-3.3697616577,-0.000012406,-1.7972270928| \mathrm{C},-1.247144804,-0.0$ $000179167,-2.1263305666|\mathrm{H},-1.3537809142,-0.0000340538,-3.1932507938| \mathrm{C}$, $0.0216758649,0.0000027961,-1.5939111732 \mid \mathrm{C}, 1.1240950223,0.0000499365,-2$ $.5406261696|\mathrm{H}, 0.8520428904,-0.0000998015,-3.5900665832| \mathrm{C}, 0.1902470991$, $0.0000072272,-0.1545169676|\mathrm{C}, 2.8210490175,-0.0000220831,-0.0899028324|$ $\mathrm{H}, 2.7978884839,0.0001448551,-1.160856176 \mid \mathrm{H}, 3.3740762924,-0.8744385166$, $0.2350331203|\mathrm{H}, 3.374222809,0.8742004335,0.2353018879| \mid$ Version=x86-Win3 2-G98RevA. $11.4|\mathrm{HF}=-459.47651| \mathrm{S} 2=0.761031|\mathrm{~S} 2-1=0.| \mathrm{S} 2 \mathrm{~A}=0.022673 \mid \mathrm{RMSD}=3.9$ $01 e-009|\mathrm{RMSF}=6.430 \mathrm{e}-005| \mathrm{Dipole}=-0.6175273,-0.000092,0.0940591 \mid \mathrm{PG}=\mathrm{C} 01 \quad[$ $\mathrm{X}(\mathrm{C} 12 \mathrm{H} 10)]||[$

\section{UCCSD(T)/4-31G}

1 | 1 | UNPC-UNK | SP | UCCSD ( T) -RW | 4-31G |C12H10|PCUSER | 10-Apr-2003|0||\#UCCSD ( $\mathrm{T}, \mathrm{WINDOW}=(27,124)) / 4-31 \mathrm{G}$ GUESS $=($ ALWAYS, $\mathrm{MIX})||$ molecule 38|| $0,1|\mathrm{C}| \mathrm{C}, 1,1$. $36874726|\mathrm{H}, 2,1.0732113,1,118.28597352| \mathrm{C}, 2,1.40018199,1,123.20566419,3$, 180 , $0 \mid \mathrm{H}, 4,1.07168353,2,119.82888025,1,180$, $0 \mid \mathrm{C}, 4,1.35345998,2,119.161$ $93434,1,0.0001153,0|\mathrm{H}, 6,1.07238635,4,120.98772242,2,179.99988314,0| \mathrm{C}, 6$ ,1.41176329,4,120.37663605,2,0 . 0|C,8,1.417037,6,118.27037661,4,179.99 $9737,0|\mathrm{H}, 9,1.0726367,8,118.03598401,6,0.00027693,0| \mathrm{C}, 9,1.35270575,8,12$ $1.50108083,6,-179.99936036,0 \mid \mathrm{H}, 11,1.07112922,9,121.16200231,8,179.9996$ $7626,0|\mathrm{C}, 11,1.40083074,9,118.75647957,8,-0.00010584,0| \mathrm{H}, 13,1.072236,11$ $, 118.66876483,9,179.9996719,0 \mid C, 13,1.37600011,11,122.85983974,9,-0.000$ 
$91964,0|\mathrm{C}, 15,1.45313361,13,116.58151421,11,-179.99724951,0| \mathrm{H}, 16,1.0841$ $2987,15,116.12153456,13,-0.00969086,0 \mid C, 15,1.4492315,13,119.44340736,1$ $1,0.00138931,0|\mathrm{C}, 1,1.51816741,18,125.9600111,8,179.99910726,0| \mathrm{H}, 19,1.0$ $7120376,1,113.55711509,18,0.0102577,0 \mid \mathrm{H}, 19,1.08444762,1,109.71219818,1$ $8,-121.0726219,0|\mathrm{H}, 19,1.08444657,1,109.71199509,18,121.09296774,0| \mid$ Ver sion=x86-Win32-G98RevA.11.4|HF=-459.47651|MP 2=-459.8909503|MP 3=-459.91 $74735|\mathrm{MP} 4 \mathrm{D}=-459.9292399| \mathrm{MP} 4 \mathrm{DQ}=-459.9200913|\mathrm{PUHF}=-459.4683573| \mathrm{PMP} 2-0=-4$ $59.88268|\mathrm{PMP} 3-0=-459.9091423| \mathrm{MP} 4 \mathrm{SDQ}=-459.9245282|\mathrm{CCSD}=-459.9317386| \mathrm{CCS}$ $\mathrm{D}(\mathrm{T})=-459.9545503|\mathrm{~S} 2=0.761031| \mathrm{S} 2-1=0.783302|\mathrm{~S} 2 \mathrm{~A}=0.022673| \mathrm{RMSD}=8.598 \mathrm{e}-0$ $09|\mathrm{PG}=\mathrm{C} 01 \quad[\mathrm{X}(\mathrm{C} 12 \mathrm{H} 10)]| \mid @$

\section{UB3LYP/4-31G}

1 | 1 | UNPC-UNK | SP | UB3LYP | 4-31G | C12H10 | PCUSER | 31-Jan-2003 | 0 || \#UB3LYP / 4-31 G GUESS $=($ ALWAYS, MIX) || molecule 38||0,1|C|C, 1, 1.36874726|H, 2, 1.0732113, $1,118.28597352|\mathrm{C}, 2,1.40018199,1,123.20566419,3,180 ., 0| \mathrm{H}, 4,1.07168353,2$ $, 119.82888025,1,180 ., 0|\mathrm{C}, 4,1.35345998,2,119.16193434,1,0.0001153,0| \mathrm{H}, 6$ $, 1.07238635,4,120.98772242,2,179.99988314,0 \mid \mathrm{C}, 6,1.41176329,4,120.37663$ $605,2,0 ., 0|\mathrm{C}, 8,1.417037,6,118.27037661,4,179.999737,0| \mathrm{H}, 9,1.0726367,8$, $118.03598401,6,0.00027693,0 \mid C, 9,1.35270575,8,121.50108083,6,-179.99936$ $036,0|\mathrm{H}, 11,1.07112922,9,121.16200231,8,179.99967626,0| \mathrm{C}, 11,1.40083074$, $9,118.75647957,8,-0.00010584,0 \mid \mathrm{H}, 13,1.072236,11,118.66876483,9,179.999$ $6719,0|\mathrm{C}, 13,1.37600011,11,122.85983974,9,-0.00091964,0| \mathrm{C}, 15,1.45313361$ ,13,116.58151421,11,-179.99724951,0|H, 16, 1.08412987, 15, 116.12153456,13 $,-0.00969086,0|C, 15,1.4492315,13,119.44340736,11,0.00138931,0| C, 1,1.51$ $816741,18,125.9600111,8,179.99910726,0 \mid \mathrm{H}, 19,1.07120376,1,113.55711509$, $18,0.0102577,0|\mathrm{H}, 19,1.08444762,1,109.71219818,18,-121.0726219,0| \mathrm{H}, 19,1$ $.08444657,1,109.71199509,18,121.09296774,0||$ Version=x86-Win32-G98RevA. $11.4|\mathrm{HF}=-462.5713673| \mathrm{S} 2=0.696853|\mathrm{~S} 2-1=0.| \mathrm{S} 2 \mathrm{~A}=0.007116|\mathrm{RMSD}=1.571 \mathrm{e}-005|$ Dipole $=-0.7545681,-0.0001284,0.3141184 \mid \mathrm{PG}=\mathrm{C} 01$ [X (C12H10) ] | @

\section{UHF/6-311G(d)}

1 | 1 | UNPC-UNK | FOpt | UHF | 6-311G (d) |C12H10|PCUSER|05-Dec-2003|0||\#P UHF/6$311 \mathrm{G}$ (D) GUESS=READ OPT SCF (CONVER=5) GEOM=CHECKPOINT ||mol6-rohf-sto-3g1|| $0,1|C, 1.4562429255,0.0001066688,0.5392087921| C, 1.4388990005,0.00009$ $70988,1.9104942396|\mathrm{H}, 2.3841464153,0.0000952308,2.4255601789| \mathrm{C}, 0.265373$ $8394,0.0000719022,2.6783067747 \mid \mathrm{H}, 0.3245709212,0.0000607523,3.751599260$ $3|\mathrm{C},-0.929942587,0.0000464216,2.0422965852| \mathrm{H},-1.8520172066,0.000016993$ $4,2.5955467749|\mathrm{C},-0.9790286713,0.0000262752,0.6296281006| \mathrm{C},-2.26091805$ $47,-0.0000547119,0.0183052387 \mid \mathrm{H},-3.1231014693,-0.0000542925,0.66194699$ $07|\mathrm{C},-2.4230425765,-0.0001616198,-1.3280453174| \mathrm{H},-3.4000602974,-0.0002$ $357477,-1.7741716296|\mathrm{C},-1.2717692112,-0.0001875483,-2.1243914509| \mathrm{H},-1$. $3877492435,-0.0002736107,-3.1924739895 \mid \mathrm{C}, 0.0171223235,-0.0000288978,-1$ $.6133956082|\mathrm{C}, 1.1017539162,0.0001814621,-2.5630332309| \mathrm{H}, 0.6486553339,-$ $0.0005317364,-3.5557309087|\mathrm{C}, 0.2025155636,0.00006434,-0.1612277649| \mathrm{C}, 2$ $.8464035397,0.0000210179,-0.0773467168 \mid \mathrm{H}, 2.8402665658,0.0015174474,-1$. $1474247416|\mathrm{H}, 3.3912104365,-0.8753402383,0.2639383888| \mathrm{H}, 3.3924184982,0$. $8736507488,0.2664118245||$ Version=x86-Win32-G98RevA.11.4|HF=-460.201567 $2|\mathrm{~S} 2=0.| \mathrm{S} 2-1=0 .|\mathrm{S} 2 \mathrm{~A}=0.| \mathrm{RMSD}=2.549 \mathrm{e}-006|\mathrm{RMSF}=8.772 \mathrm{e}-005| \mathrm{Dipole}=-1.26235$ $78,-0.0020328,0.4596219|\mathrm{PG}=\mathrm{C} 01 \quad[\mathrm{X}(\mathrm{C} 12 \mathrm{H} 10)]| \mid \mathrm{Q}$

\section{UB3LYP/6-311G(d)}


$1|1|$ UNPC-UNK | SP |UB3LYP | 6-311G (d) |C12H10|PCUSER |05-DeC-2003|0||\#P UB3LY P /6-311G (D) GUESS=READ SP SCF (CONVER=5) GEOM=CHECKPOINT || mol6-ub-6-311g $\mathrm{d}-1|| 0,1|\mathrm{C}, 1.4562429255,0.0001066688,0.5392087921| \mathrm{C}, 1.4388990005,0.000$ $0970988,1.9104942396|\mathrm{H}, 2.3841464153,0.0000952308,2.4255601789| \mathrm{C}, 0.2653$ $738394,0.0000719022,2.6783067747 \mid \mathrm{H}, 0.3245709212,0.0000607523,3.7515992$ $603|\mathrm{C},-0.929942587,0.0000464216,2.0422965852| \mathrm{H},-1.8520172066,0.0000169$ $934,2.5955467749|\mathrm{C},-0.9790286713,0.0000262752,0.6296281006| \mathrm{C},-2.260918$ $0547,-0.0000547119,0.0183052387 \mid \mathrm{H},-3.1231014693,-0.0000542925,0.661946$ $9907|\mathrm{C},-2.4230425765,-0.0001616198,-1.3280453174| \mathrm{H},-3.4000602974,-0.00$ $02357477,-1.7741716296|\mathrm{C},-1.2717692112,-0.0001875483,-2.1243914509| \mathrm{H},-$ $1.3877492435,-0.0002736107,-3.1924739895 \mid C, 0.0171223235,-0.0000288978$, $-1.6133956082|\mathrm{C}, 1.1017539162,0.0001814621,-2.5630332309| \mathrm{H}, 0.6486553339$ $,-0.0005317364,-3.5557309087|C, 0.2025155636,0.00006434,-0.1612277649| \mathrm{C}$ , $2.8464035397,0.0000210179,-0.0773467168 \mid \mathrm{H}, 2.8402665658,0.0015174474$,$1.1474247416|\mathrm{H}, 3.3912104365,-0.8753402383,0.2639383888| \mathrm{H}, 3.3924184982$, $0.8736507488,0.2664118245||$ Version $=x 86-W i n 32-G 98 R e v A .11 .4 \mid \mathrm{HF}=-463.2630$ $819|\mathrm{~S} 2=0.| \mathrm{S} 2-1=0 .|\mathrm{S} 2 \mathrm{~A}=0.| \mathrm{RMSD}=9.138 \mathrm{e}-006 \mid \mathrm{Dipole}=-1.3251567,-0.0006597$, $0.7322935|\mathrm{PG}=\mathrm{C} 01 \quad[\mathrm{X}(\mathrm{C} 12 \mathrm{H} 10)]| \mid \varrho$

\section{RHF/STO-3G}

1 1 1 | UNPC-UNK $\mid$ SP $\mid$ ROHF $\mid$ STO-3G $\mid$ C12H10|PCUSER 0 05-DeC-2003|0||\#P ROHF/STO-3 G SCF (CONVER=5)||mol6-rohf-sto-3g-1||0,1|C|C,1,1.36874726|H, 2, 1.073211 $3,1,118.28597352|\mathrm{C}, 2,1.40018199,1,123.20566419,3,180 ., 0| \mathrm{H}, 4,1.07168353$ $, 2,119.82888025,1,180 ., 0|\mathrm{C}, 4,1.35345998,2,119.16193434,1,0.0001153,0| \mathrm{H}$ $, 6,1.07238635,4,120.98772242,2,179.99988314,0 \mid C, 6,1.41176329,4,120.376$ $63605,2,0 ., 0|\mathrm{C}, 8,1.417037,6,118.27037661,4,179.999737,0| \mathrm{H}, 9,1.0726367$, $8,118.03598401,6,0.00027693,0 \mid C, 9,1.35270575,8,121.50108083,6,-179.999$ $36036,0|\mathrm{H}, 11,1.07112922,9,121.16200231,8,179.99967626,0| \mathrm{C}, 11,1.4008307$ $4,9,118.75647957,8,-0.00010584,0 \mid \mathrm{H}, 13,1.072236,11,118.66876483,9,179.9$ $996719,0|C, 13,1.37600011,11,122.85983974,9,-0.00091964,0| C, 15,1.453133$ $61,13,116.58151421,11,-179.99724951,0 \mid \mathrm{H}, 16,1.08412987,15,116.12153456$, $13,-0.00969086,0|C, 15,1.4492315,13,119.44340736,11,0.00138931,0| C, 1,1$. $51816741,18,125.9600111,8,179.99910726,0 \mid \mathrm{H}, 19,1.07120376,1,113.5571150$ $9,18,0.0102577,0|\mathrm{H}, 19,1.08444762,1,109.71219818,18,-121.0726219,0| \mathrm{H}, 19$ ,1.08444657,1,109.71199509,18,121.09296774,0||Version=x86-Win32-G98Rev A.11.4 $|\mathrm{HF}=-454.5054628| \mathrm{RMSD}=1.370 \mathrm{e}-006 \mid \mathrm{Dipole}=-0.9060904,-0.0001138,0$. $1526284|\mathrm{PG}=\mathrm{C} 01 \quad[\mathrm{X}(\mathrm{C} 12 \mathrm{H} 10)]| \mid$ e

\section{RHF/6-311G(d)}

1 | 1 |UNPC-UNK |SP $\mid$ ROHF |6-311G (d) |C12H10|PCUSER |05-Dec-2003|0||\#P ROHF /6$311 \mathrm{G}(\mathrm{D})$ GUESS=READ SCF (CONVER=5) GEOM=CHECKPOINT $\mid$ mol6-rohf-sto-3g-1 ||0 $, 1|\mathrm{C}| \mathrm{C}, 1,1.36874726|\mathrm{H}, 2,1.0732113,1,118.28597352| \mathrm{C}, 2,1.40018199,1,123$. $20566419,3,180 ., 0|\mathrm{H}, 4,1.07168353,2,119.82888025,1,180 ., 0| \mathrm{C}, 4,1.3534599$ $8,2,119.16193434,1,0.0001153,0 \mid \mathrm{H}, 6,1.07238635,4,120.98772242,2,179.999$ $88314,0|C, 6,1.41176329,4,120.37663605,2,0 ., 0| C, 8,1.417037,6,118.270376$ $61,4,179.999737,0|\mathrm{H}, 9,1.0726367,8,118.03598401,6,0.00027693,0| \mathrm{C}, 9,1.35$ $270575,8,121.50108083,6,-179.99936036,0 \mid \mathrm{H}, 11,1.07112922,9,121.16200231$ , 8,179.99967626,0|C,11,1.40083074,9,118.75647957,8,-0.00010584, 0| H, 13, $1.072236,11,118.66876483,9,179.9996719,0 \mid C, 13,1.37600011,11,122.859839$ $74,9,-0.00091964,0|C, 15,1.45313361,13,116.58151421,11,-179.99724951,0|$ $\mathrm{H}, 16,1.08412987,15,116.12153456,13,-0.00969086,0 \mid \mathrm{C}, 15,1.4492315,13,119$ $.44340736,11,0.00138931,0 \mid C, 1,1.51816741,18,125.9600111,8,179.99910726$ , $0|\mathrm{H}, 19,1.07120376,1,113.55711509,18,0.0102577,0| \mathrm{H}, 19,1.08444762,1,109$ 
$.71219818,18,-121.0726219,0 \mid \mathrm{H}, 19,1.08444657,1,109.71199509,18,121.0929$ $6774,0||$ Version=x86-Win32-G98RevA.11.4|HF=-460.1974717|RMSD=5.539e-006

|Dipole $=-1.1985837,-0.0001301,0.3051234 \mid \mathrm{PG}=\mathrm{C} 01$ [X(C12H10)] || @

\section{RHF/6-311G(d,p)}

1 | 1 | UNPC-UNK | FOpt |ROHF |6-311G (d, p) |C12H10|PCUSER|08-Dec-2003|0||\#P ROH F/6-311** OPT GUESS=READ SCF (CONVER=5) GEOM=CHECKPOINT || $\operatorname{mol} 6|| 0,1 \mid \mathrm{C}, 1$. $4565193239,-0.0001795968,0.5400381159 \mid C, 1.4387290972,-0.0009201043,1.9$ $113028043|\mathrm{H}, 2.3840729637,-0.0014438696,2.4264621133| \mathrm{C}, 0.264775011,-0.0$ $011251948,2.6783608528|\mathrm{H}, 0.3236704231,-0.0017561277,3.7517778121| \mathrm{C},-0$. $9303372865,-0.0006163067,2.0423936043 \mid \mathrm{H},-1.8532392396,-0.0008601105,2$. $5943849596|\mathrm{C},-0.9789130684,0.0000838729,0.6297240965| \mathrm{C},-2.260744041,0$. $0002129309,0.0181703912|\mathrm{H},-3.1224169897,-0.0000551119,0.662923658| \mathrm{C},-2$ $.4226371338,0.0005061834,-1.3279732757 \mid \mathrm{H},-3.3996358335,0.000515991,-1$. $7743564545|\mathrm{C},-1.2711467503,0.0007507141,-2.1242005529| \mathrm{H},-1.3852604008$, $0.0009119153,-3.1926901317|\mathrm{C}, 0.0178113915,0.0008177539,-1.6128770595| \mathrm{C}$ $, 1.1028801154,0.0010577745,-2.5626036001 \mid \mathrm{H}, 0.6508054067,-0.0010162712$, $-3.5562918056|\mathrm{C}, 0.2028558351,0.0004126711,-0.1606503502| \mathrm{C}, 2.8455380165$ $,-0.0003265654,-0.0797050006 \mid \mathrm{H}, 2.8311207182,-0.0092191631,-1.151054501$ $4|\mathrm{H}, 3.3925859878,-0.8717433275,0.2706862225| \mathrm{H}, 3.3863139001,0.880621277$ 3,0.2562779719||Version=x86-Win32-G98RevA.11.4|HF=-460.2188294|RMSD=1. $332 e-006|\mathrm{RMSF}=4.413 e-005| \mathrm{Dipole}=-1.2474624,-0.0007853,0.4445283 \mid \mathrm{PG}=\mathrm{C} 01$ $[\mathrm{X}(\mathrm{C} 12 \mathrm{H} 10)]|| @$

\section{RB3LYP/6-311G(d,p)}

1 | 1 | UNPC-UNK | FOpt |ROB3LYP |6-311G (d, p) |C12H10|PCUSER |09-DeC-2003|0|| \#P ROB3LYP/6-311** OPT GUESS=READ SCF (CONVER=5) GEOM=CHECKPOINT || mol6||0, $1|C, 1.4610774791,-0.0003026603,0.5397297533| C, 1.4429074141,-0.00053011$ $83,1.9296995816|\mathrm{H}, 2.3983021284,-0.0009412399,2.4455592283| \mathrm{C}, 0.26770637$ $21,-0.0003922717,2.6949740163 \mid \mathrm{H}, 0.3249538074,-0.0005569387,3.777529087$ $1|\mathrm{C},-0.9421279283,-0.0001814409,2.0530118616| \mathrm{H},-1.8728461866,-0.000171$ $9569,2.6093484356|\mathrm{C},-0.9920516074,-0.0000674163,0.6377064267| \mathrm{C},-2.2676$ $9835,0.0000084932,0.0208773897 \mid \mathrm{H},-3.1400941953,-0.0000441137,0.6669986$ $984|\mathrm{C},-2.4253181029,0.0000244982,-1.3456961182| \mathrm{H},-3.411988234,0.000022$ $4278,-1.7935996326|\mathrm{C},-1.2783014126,-0.0000848959,-2.1393196306| \mathrm{H},-1.38$ $15981185,-0.0001018047,-3.2187314045 \mid \mathrm{C}, 0.0345503774,0.0002823393,-1.62$ $18215404|\mathrm{C}, 1.1251911446,0.0023727163,-2.54946471| \mathrm{H}, 0.6687700826,0.0004$ $230678,-3.5572503735|\mathrm{C}, 0.2108716035,0.0000061407,-0.1609577212| \mathrm{C}, 2.836$ $9486607,-0.0007091574,-0.0964117064 \mid \mathrm{H}, 2.7825979925,-0.005600034,-1.180$ $9528388|\mathrm{H}, 3.3965177782,-0.879060906,0.2430276041| \mathrm{H}, 3.3928510436,0.8834$ $74136,0.2341055808||$ Version=x86-Win32-G98RevA.11.4|HF=-463.2822824|RMS $\mathrm{D}=3.553 e-006|\mathrm{RMSF}=1.195 \mathrm{e}-004| \mathrm{Dipole}=-1.3479327,-0.0025317,0.7668861 \mid \mathrm{PG}$ $=\mathrm{C} 01 \quad[\mathrm{X}(\mathrm{C} 12 \mathrm{H} 10)]|| \mathrm{e}$

\section{Triplet}

\section{UHF/STO-3G}

1 | 1 | UNPC-UNK |FOpt | UHF |STO-3G |C12H10 (3) |PCUSER|19-OCt-2002|0||\#UHF STO3G OPT GUESS=(ALWAYS, MIX) || molecule 38||0,3|C,1.4407185058,-0.00009994 $9,0.5944012937|\mathrm{C}, 1.4079859284,-0.0001334305,2.015907349| \mathrm{H}, 2.3477919272$ 
$,-0.0001941257,2.5532469145|C, 0.1955792843,-0.0001053574,2.7424917835|$ $\mathrm{H}, 0.2092916717,-0.0001373684,3.8247959149 \mid \mathrm{C},-1.0228112202,-0.000048125$ $7,2.0444737881|\mathrm{H},-1.9625966321,-0.00003616,2.5814261013| \mathrm{C},-1.047955381$ $8,0.0000015031,0.6106205044 \mid \mathrm{C},-2.3171862857,0.0000624761,-0.0630062421$ $|\mathrm{H},-3.2205593887,0.0000589035,0.5332558281| \mathrm{C},-2.3957081323,0.000126755$ $3,-1.4692008148|\mathrm{H},-3.3591727519,0.0001710043,-1.9627433552| \mathrm{C},-1.211466$ $1218,0.0001460994,-2.2322723937 \mid \mathrm{H},-1.268338536,0.0002111968,-3.3134198$ $682|C, 0.0984056129,0.0000921727,-1.618208079| C, 1.2155506329,0.00016301$ $12,-2.4856236439|\mathrm{H}, 1.2151918483,0.0002436593,-3.5686899428| \mathrm{C}, 0.1971227$ $499,-0.0000066608,-0.1483802768 \mid \mathrm{C}, 2.8428383758,-0.0001625918,-0.019881$ $7134|\mathrm{H}, 2.8351542962,-0.0004317816,-1.1008825294| \mathrm{H}, 3.3924437231,-0.8774$ $241326,0.3127568346|\mathrm{H}, 3.3923501527,0.8773233884,0.3123247718| \mid$ Version $=$ $\mathrm{x} 86-$ Win32-G98RevA.11.4 $|\mathrm{HF}=-454.6476245| \mathrm{S} 2=3.906689|\mathrm{~S} 2-1=0.| \mathrm{S} 2 \mathrm{~A}=5.74766$ $3|\mathrm{RMSD}=8.281 \mathrm{e}-009| \mathrm{RMSF}=1.055 \mathrm{e}-005 \mid \mathrm{Dipole}=0.0003039,0.0000065,-0.069710$ $1|\mathrm{PG}=\mathrm{C} 01 \quad[\mathrm{X}(\mathrm{C} 12 \mathrm{H} 10)]| \mid \Theta$

\section{UCCSD(T)/STO-3G}

$1|1|$ UNPC-UNK $\mid$ SP $\mid$ UCCSD ( T) -FC $\mid$ STO-3G|C12H10 (3) |PCUSER $|21-O C t-2002| 0||$ \#UC CSD-T/STO-3G GUESS= (ALWAYS, MIX) || molecule 38||0,3|C|C,1,1.42188287|H, 2 $, 1.08257523,1,118.43991569 \mid \mathrm{C}, 2,1.41345492,1,122.25297801,3,-179.999274$ $46,0|\mathrm{H}, 4,1.08239099,2,120.20800184,1,179.99969014,0| \mathrm{C}, 4,1.40417397,2,1$ $19.25765219,1,0.00027416,0 \mid \mathrm{H}, 6,1.08236519,4,120.44977175,2,179.9993657$ $8,0|C, 6,1.43407373,4,120.81310901,2,-0.00091204,0| C, 8,1.43691339,6,118$ $.96114941,4,-179.99985508,0 \mid \mathrm{H}, 9,1.08241001,8,118.61709707,6,-0.0006212$ $2,0|\mathrm{C}, 9,1.4083852,8,121.15258401,6,179.99933474,0| \mathrm{H}, 11,1.08251943,9,12$ $0.32023597,8,179.99992361,0 \mid C, 11,1.40879643,9,119.59981204,8,-0.000679$ $48,0|\mathrm{H}, 13,1.08264229,11,119.78468927,9,-179.99904138,0| \mathrm{C}, 13,1.44666477$ $, 11,122.087057,9,0.00045357,0 \mid C, 15,1.41436302,13,117.0551258,11,-179.9$ $9795457,0|\mathrm{H}, 16,1.08306636,15,127.80883779,13,-0.00069043,0| \mathrm{C}, 15,1.4731$ $3911,13,118.95940681,11,0.00094374,0 \mid C, 1,1.5307788,18,125.4920293,8,17$ $9.9981699,0|\mathrm{H}, 19,1.08102816,1,113.25144693,18,-0.01738125,0| \mathrm{H}, 19,1.087$ $33723,1,109.89197655,18,-120.91294649,0 \mid \mathrm{H}, 19,1.08733895,1,109.89283297$ $, 18,120.8790617,0||$ Version=x86-Win32-G98RevA.11.4 $|\mathrm{HF}=-454.6476245| \mathrm{MP} 2=$ $-455.1559538|\mathrm{MP} 3=-455.2529159| \mathrm{MP} 4 \mathrm{D}=-455.2840227|\mathrm{MP} 4 \mathrm{DQ}=-455.2791726| \mathrm{PUH}$ $\mathrm{F}=-454.7853598|\mathrm{PMP} 2-0=-455.2882201| \mathrm{PMP} 3-0=-455.3767887 \mid \mathrm{MP} 4 \mathrm{SDQ}=-455.291$ $5732|\mathrm{CCSD}=-455.3603102| \operatorname{CCSD}(\mathrm{T})=-455.3788491|\mathrm{~S} 2=3.906689| \mathrm{S} 2-1=3.541019 \mid$ $\mathrm{S} 2 \mathrm{~A}=5.747661|\mathrm{RMSD}=3.002 \mathrm{e}-009| \mathrm{PG}=\mathrm{C} 01 \quad[\mathrm{X}(\mathrm{C} 12 \mathrm{H} 10)]|| \mathrm{a}$

\section{UB3LYP/STO-3G}

1 | 1 | UNPC-UNK |SP | UB3LYP |STO-3G|C12H10 (3) |PCUSER $02-F e b-2003|0| \mid$ \#UB3LYP / STO-3G GUESS=(ALWAYS, MIX) || molecule 38||0,3|C|C, 1, 1.42188287| H, 2, 1.082 $57523,1,118.43991569|\mathrm{C}, 2,1.41345492,1,122.25297801,3,-179.99927446,0| \mathrm{H}$ $, 4,1.08239099,2,120.20800184,1,179.99969014,0 \mid C, 4,1.40417397,2,119.257$ $65219,1,0.00027416,0|\mathrm{H}, 6,1.08236519,4,120.44977175,2,179.99936578,0| \mathrm{C}$, $6,1.43407373,4,120.81310901,2,-0.00091204,0 \mid C, 8,1.43691339,6,118.96114$ $941,4,-179.99985508,0|\mathrm{H}, 9,1.08241001,8,118.61709707,6,-0.00062122,0| \mathrm{C}$, $9,1.4083852,8,121.15258401,6,179.99933474,0 \mid \mathrm{H}, 11,1.08251943,9,120.3202$ $3597,8,179.99992361,0|\mathrm{C}, 11,1.40879643,9,119.59981204,8,-0.00067948,0| \mathrm{H}$ $, 13,1.08264229,11,119.78468927,9,-179.99904138,0 \mid C, 13,1.44666477,11,12$ $2.087057,9,0.00045357,0 \mid C, 15,1.41436302,13,117.0551258,11,-179.9979545$ $7,0|\mathrm{H}, 16,1.08306636,15,127.80883779,13,-0.00069043,0| \mathrm{C}, 15,1.47313911,1$ 
$3,118.95940681,11,0.00094374,0 \mid C, 1,1.5307788,18,125.4920293,8,179.9981$ $699,0|\mathrm{H}, 19,1.08102816,1,113.25144693,18,-0.01738125,0| \mathrm{H}, 19,1.08733723$, $1,109.89197655,18,-120.91294649,0 \mid \mathrm{H}, 19,1.08733895,1,109.89283297,18,12$ $0.8790617,0||$ Version=x86-Win32-G98RevA.11.4|HF=-457.5171094|S2=2.09333 $3|\mathrm{~S} 2-1=0.| \mathrm{S} 2 \mathrm{~A}=2.003736|\mathrm{RMSD}=8.804 \mathrm{e}-005| \mathrm{Dipole}=0.0631247,0.0000113,-0.1$ $172089|\mathrm{PG}=\mathrm{C} 01 \quad[\mathrm{X}(\mathrm{C} 12 \mathrm{H} 10)]| \mid \mathrm{e}$

\section{UHF/4-31G}

1 | 1 | UNPC-UNK |FOpt |UHF | 4-31G|C12H10 (3) |PCUSER |02-Dec-2002|0||\#UHF 4-31G OPT GUESS $=($ ALWAYS, MIX) || molecule 38|| $0,3 \mid \mathrm{C}, 1.4343940179,-0.0304369014$ $, 0.5652262986|\mathrm{C}, 1.4306053048,-0.0726765093,1.9623633807| \mathrm{H}, 2.3697829897$ $,-0.0968215228,2.4794047905|\mathrm{C}, 0.2439783141,-0.0843705125,2.7043476018|$ $\mathrm{H}, 0.2817580973,-0.1171118675,3.7751480365 \mid \mathrm{C},-0.9713439655,-0.053197679$ $7,2.0456261493|\mathrm{H},-1.8905732642,-0.0614393933,2.5986231036| \mathrm{C},-1.0279849$ $442,-0.0097956042,0.6322627325 \mid C,-2.2908022439,0.0214786327,-0.0155617$ $787|\mathrm{H},-3.1779015522,0.0113339884,0.5873391087| \mathrm{C},-2.3858094475,0.064312$ $3624,-1.4014237573|\mathrm{H},-3.3466825829,0.0874607817,-1.8762721065| \mathrm{C},-1.229$ $5484871,0.0771678325,-2.1743792182 \mid \mathrm{H},-1.3029877854,0.1102271505,-3.244$ $0159865|C, 0.0732409958,0.0476355751,-1.5872921348| C, 1.1659669135,0.063$ $7886133,-2.4512691259|\mathrm{H}, 1.2305416943,0.0956079179,-3.5186035016| \mathrm{C}, 0.18$ $86948284,0.0022740037,-0.1364977776 \mid \mathrm{C}, 2.7766570285,-0.0214079919,-0.12$ $70870448|\mathrm{H}, 2.9008174119,-0.8762258501,-0.7799234612| \mathrm{H}, 3.5704811917,-0$. $0509214243,0.6070718783|\mathrm{H}, 2.9164739105,0.8692592957,-0.7266638149| \mid$ Ver sion $=x 86-W i n 32-G 98 R e v A .11 .4|\mathrm{HF}=-459.5485481| \mathrm{S} 2=3.271102|\mathrm{~S} 2-1=0.| \mathrm{S} 2 \mathrm{~A}=3$. $472714|\mathrm{RMSD}=5.253 \mathrm{e}-009| \mathrm{RMSF}=1.995 \mathrm{e}-005 \mid \mathrm{Dipole}=-0.1043131,0.0041884,-0$. $1018792 \mid \mathrm{PG}=\mathrm{C} 01 \quad[\mathrm{X}(\mathrm{C} 12 \mathrm{H} 10)]$ || @

\section{$\mathrm{UCCSD}(\mathrm{T}) / 4-31 G$}

$1|1|$ UNPC-UNK | SP |UCCSD (T) -RW | 4-31G|C12H10 (3) |PCUSER |09-Apr-2003|0|| \#UCC $\operatorname{SD}(\mathrm{T}, \mathrm{WINDOW}=(27,124)) / 4-31 \mathrm{G}$ GUESS $=($ ALWAYS, MIX $)||$ molecule 38 || $0,3|\mathrm{C}| \mathrm{C}, 1$ $, 1.39778059|\mathrm{H}, 2,1.07236632,1,118.70072188| \mathrm{C}, 2,1.39955741,1,122.1720141$ $2,3,179.99921318,0|\mathrm{H}, 4,1.07196683,2,119.99690587,1,180 ., 0| \mathrm{C}, 4,1.382712$ $53,2,119.50264635,1,-0.01069699,0 \mid \mathrm{H}, 6,1.0727796,4,120.49000716,2,-179$. $99043078,0|C, 6,1.41516363,4,120.77462505,2,0.00498547,0| C, 8,1.41963453$ , 6, 119.47410539,4,179.99975898,0| H, 9,1.07263115,8,118.61833771,6,-0.00 $092911,0|\mathrm{C}, 9,1.38977499,8,121.10019003,6,-179.99581429,0| \mathrm{H}, 11,1.072051$ $3,9,120.24010638,8,179.99377341,0 \mid C, 11,1.39088634,9,119.84260251,8,-0$. $00627513,0|\mathrm{H}, 13,1.07266447,11,119.8366993,9,179.99989869,0| \mathrm{C}, 13,1.4292$ $6689,11,121.95722084,9,-0.00634149,0 \mid C, 15,1.39311417,13,117.38595272,1$ $1,179.99878555,0|\mathrm{H}, 16,1.06975934,15,131.79471862,13,0.01457718,0| \mathrm{C}, 15$, $1.45608775,13,118.82822948,11,0.02315155,0 \mid C, 1,1.5103143,18,123.302969$ $05,8,-179.98050705,0|\mathrm{H}, 19,1.08273947,1,111.9365162,18,-60.39224464,0| \mathrm{H}$ $, 19,1.08167332,1,109.93430356,18,179.99413548,0 \mid \mathrm{H}, 19,1.08274156,1,111$. $93279283,18,60.38388763,0||$ Version=x86-Win32-G98RevA.11.4 HF=-459.5485 $481|\mathrm{MP} 2=-459.8864912| \mathrm{MP} 3=-459.9169006|\mathrm{MP} 4 \mathrm{D}=-459.9256565| \mathrm{MP} 4 \mathrm{DQ}=-459.921$ $0558|\mathrm{PUHF}=-459.6061049| \mathrm{PMP} 2-0=-459.940733|\mathrm{PMP} 3-0=-459.9662972| \mathrm{MP} 4 \mathrm{SDQ}=-$ $459.9291156|\operatorname{CCSD}=-459.9536683| \operatorname{CCSD}(\mathrm{T})=-459.9719284|\mathrm{~S} 2=3.271101| \mathrm{S} 2-1=3$. $021819|\mathrm{~S} 2 \mathrm{~A}=3.472713| \mathrm{RMSD}=7.191 \mathrm{e}-009|\mathrm{PG}=\mathrm{C} 01 \quad[\mathrm{X}(\mathrm{C} 12 \mathrm{H} 10)]| \mid \mathrm{e}$

\section{UB3LYP/4-31G}


$1|1|$ UNPC-UNK | SP |UB3LYP | 4-31G |C12H10 (3) |PCUSER | 31-Jan-2003|0|| \#UB3LYP / 4 -31G GUESS=(ALWAYS, MIX) || molecule 38||0,3|C|C,1,1.39778059| H, 2, 1.07236 $632,1,118.70072188|\mathrm{C}, 2,1.39955741,1,122.17201412,3,179.99921318,0| \mathrm{H}, 4$, $1.07196683,2,119.99690587,1,180 ., 0 \mid C, 4,1.38271253,2,119.50264635,1,-0$. $01069699,0|\mathrm{H}, 6,1.0727796,4,120.49000716,2,-179.99043078,0| \mathrm{C}, 6,1.415163$ $63,4,120.77462505,2,0.00498547,0 \mid C, 8,1.41963453,6,119.47410539,4,179.9$ $9975898,0|\mathrm{H}, 9,1.07263115,8,118.61833771,6,-0.00092911,0| \mathrm{C}, 9,1.38977499$ ,8,121.10019003,6,-179.99581429,0|H,11,1.0720513,9,120.24010638,8,179. $99377341,0|\mathrm{C}, 11,1.39088634,9,119.84260251,8,-0.00627513,0| \mathrm{H}, 13,1.07266$ $447,11,119.8366993,9,179.99989869,0 \mid C, 13,1.42926689,11,121.95722084,9$, $-0.00634149,0|\mathrm{C}, 15,1.39311417,13,117.38595272,11,179.99878555,0| \mathrm{H}, 16,1$ $.06975934,15,131.79471862,13,0.01457718,0 \mid C, 15,1.45608775,13,118.82822$ $948,11,0.02315155,0|\mathrm{C}, 1,1.5103143,18,123.30296905,8,-179.98050705,0| \mathrm{H}$, $19,1.08273947,1,111.9365162,18,-60.39224464,0 \mid \mathrm{H}, 19,1.08167332,1,109.93$ $430356,18,179.99413548,0 \mid \mathrm{H}, 19,1.08274156,1,111.93279283,18,60.38388763$ , 0|| Version=x86-Win32-G98RevA.11.4 $|\mathrm{HF}=-462.5909737| \mathrm{S} 2=2.053743 \mid \mathrm{S} 2-1=0$. $|\mathrm{S} 2 \mathrm{~A}=2.001156| \mathrm{RMSD}=7.161 \mathrm{e}-005|\mathrm{Dipole}=-0.0934566,0.0001484,-0.0709284| \mathrm{P}$ $\mathrm{G}=\mathrm{C} 01 \quad[\mathrm{X}(\mathrm{C} 12 \mathrm{H} 10)]|| \Theta$

\section{UHF/6-311G(d)}

1 | 1 | UNPC-UNK |FOpt | UHF | 6-311G (d) |C12H10 (3) |PCUSER 0 6-Dec-2003|0||\#P UHF /6-311G (D) GUESS=READ OPT SCF (CONVER=5) GEOM=CHECKPOINT || mol6-uhf-6-31 $1 \mathrm{gd}-3|| 0,3|\mathrm{C}, 1.4429065349,-0.000326999,0.5552109807| \mathrm{C}, 1.4474970444,-0$. $0000362887,1.9520329719|\mathrm{H}, 2.391616575,-0.0004169223,2.4666831189| \mathrm{C}, 0.2$ $646848796,0.000502576,2.7053913237 \mid \mathrm{H}, 0.3114451083,0.0007476898,3.77952$ $51054|\mathrm{C},-0.9548593304,0.0005083054,2.0556325798| \mathrm{H},-1.8718385928,0.0007$ $119585,2.6176541139|C,-1.0199654515,0.000130086,0.6405383224| C,-2.2906$ $83354,-0.0004053763,0.0028602411 \mid \mathrm{H},-3.1752994184,-0.0005796378,0.61406$ $11912|\mathrm{C},-2.3987585408,-0.0008320321,-1.3838455616| \mathrm{H},-3.3667060043,-0.0$ $01443938,-1.8519602162|\mathrm{C},-1.2485083219,-0.0002822092,-2.1670657307| \mathrm{H},-$ $1.3319623201,-0.0002848538,-3.2389620421 \mid \mathrm{C}, 0.0603198877,0.0005232079,-$ $1.5895515292|\mathrm{C}, 1.1367775029,0.0020993481,-2.4701230822| \mathrm{H}, 1.1640067187$, $0.0027974671,-3.5420222302|\mathrm{C}, 0.190336318,0.0000885108,-0.1366343716| \mathrm{C}$, $2.7822817456,-0.001386947,-0.1451846988 \mid \mathrm{H}, 2.911302979,-0.8748551234,-0$ $.7741612265|\mathrm{H}, 3.5813796063,-0.0035097906,0.5855152286| \mathrm{H}, 2.9138818604,0$ $.8733400585,-0.7719017158||$ Version=x86-Win32-G98RevA.11.4 $\mid \mathrm{HF}=-460.2737$ $071|\mathrm{~S} 2=3.192089| \mathrm{S} 2-1=0 .|\mathrm{S} 2 \mathrm{~A}=3.261787| \mathrm{RMSD}=3.167 \mathrm{e}-006|\mathrm{RMSF}=3.883 e-005| \mathrm{D}$ ipole $=-0.1218417,-0.0003021,-0.0833478|\mathrm{PG}=\mathrm{C} 01 \quad[\mathrm{X}(\mathrm{C} 12 \mathrm{H} 10)]| \mid \mathrm{e}$

\section{UB3LYP/6-311G(d)}

1 | 1 | UNPC-UNK | SP | UB3LYP | 6-311G (d) |C12H10 (3) |PCUSER 06 -Dec-2003|0||\#P UB 3LYP / 6-311G (D) GUESS=READ SCF (CONVER=5) GEOM=CHECKPOINT $\mid$ mol6-rohf-6-3 $11 \mathrm{gd}-3|| 0,3|\mathrm{C},-1.3388510233,0.7604138745,0.0000050542| \mathrm{C},-2.397086181$,$0.0992894279,-0.0000017895|\mathrm{H},-3.3953072782,0.3007568458,-0.0000043491|$ $\mathrm{C},-2.2353104286,-1.5010004232,-0.000004152 \mid \mathrm{H},-3.1032791904,-2.13582935$ $64,-0.0000082665|\mathrm{C},-0.9914214436,-2.0299720118,-0.0000012875| \mathrm{H},-0.8453$ $777621,-3.0955003982,-0.0000036065 \mid C, 0.1487615297,-1.1848823103,0.0000$ $019351|\mathrm{C}, 1.4415557028,-1.7699988754,0.0000012946| \mathrm{H}, 1.5211800629,-2.842$ $4726591,0.0000004566|\mathrm{C}, 2.552842736,-0.9960221668,0.0000019678| \mathrm{H}, 3.5323$ $363751,-1.4393760279,0.0000015934 \mid C, 2.4229794234,0.4044051331,-0.00000$ $02161|\mathrm{H}, 3.3114430204,1.0103455912,-0.0000020944| \mathrm{C}, 1.1962659533,1.01987$ 
$05078,-0.0000013824|\mathrm{C}, 1.1944207157,2.4662286699,-0.0000066088| \mathrm{H}, 2.0479$ $961067,3.1164253556,-0.0000105764 \mid C,-0.0047366644,0.2210817416,0.00000$ $45406|\mathrm{C},-1.6378681727,2.2434638556,0.0000035703| \mathrm{H},-1.2346841165,2.7399$ $980419,0.8745250762|\mathrm{H},-2.708943209,2.3998635258,-0.0000004629| \mathrm{H},-1.234$ $6768931,2.7399976789,-0.8745153279||$ Version=x86-Win32-G98RevA.11.4 $\mid \mathrm{HF}=$ $-463.2757994|\mathrm{~S} 2=2.0385| \mathrm{S} 2-1=0 .|\mathrm{S} 2 \mathrm{~A}=2.0006| \mathrm{RMSD}=7.296 \mathrm{e}-006 \mid \mathrm{Dipole}=0.118$ $6126,-0.1167686,-0.0000033|P G=\mathrm{C} 01 \quad[\mathrm{X}(\mathrm{C} 12 \mathrm{H} 10)]| \mid \varrho$

\section{ROHF/STO-3G}

1 | 1 | UNPC-UNK |FOpt |ROHF |STO-3G |C12H10 (3) |PCUSER |05-DeC-2003|0||\#P ROHF/ STO-3G OPT | mol6-rohf-sto-3g||0,3|C,1.4272527753,-0.0001231095,0.57927 $97939|\mathrm{C}, 1.4285579442,-0.0002676342,1.9386752589| \mathrm{H}, 2.3706741954,-0.0003$ $896208,2.470591386|\mathrm{C}, 0.2259190384,-0.0002667679,2.6946258276| \mathrm{H}, 0.28049$ $07583,-0.0003845924,3.7758274701 \mid C,-0.9637214914,-0.0001299892,2.05780$ $15616|\mathrm{H},-1.893942485,-0.0001326437,2.6107198121| \mathrm{C},-1.0238311257,0.0000$ $129779,0.6280451513|\mathrm{C},-2.2962688549,0.0001502689,-0.0252858277| \mathrm{H},-3.18$ $75514229,0.0001510975,0.5885005936 \mid C,-2.3805297954,0.0002857808,-1.374$ $0109127|\mathrm{H},-3.3414902258,0.000399932,-1.8725519472| \mathrm{C},-1.1956486495,0.00$ $02767352,-2.1512944976|\mathrm{H},-1.2803123307,0.0003893928,-3.2302006606| \mathrm{C}, 0$. $0439779725,0.0001248102,-1.5757002827 \mid C, 1.2172575728,0.0001242461,-2.4$ $734332717|\mathrm{H}, 1.1489666446,0.0002322752,-3.5568664391| \mathrm{C}, 0.1650486136,0.0$ $000195877,-0.1306454117|\mathrm{C}, 2.7753410978,-0.0001638328,-0.1409629344| \mathrm{H}, 2$ $.8922002195,-0.8799448968,-0.7685726141 \mid \mathrm{H}, 3.5784873324,-0.0003050678,0$ $.5883779747|\mathrm{H}, 2.892346728,0.8797256854,-0.7683923058| \mid$ Version=x86-Win3 2-G98RevA.11.4 $|\mathrm{HF}=-454.5709069| \mathrm{RMSD}=7.810 \mathrm{e}-009|\mathrm{RMSF}=3.655 \mathrm{e}-005| \mathrm{Dipole}=$ $0.0465842,0.0000009,-0.0854271|\mathrm{PG}=\mathrm{C} 01 \quad[\mathrm{X}(\mathrm{C} 12 \mathrm{H} 1 \mathrm{O})]| \mid \mathrm{a}$

\section{ROHF/6-311G(d)}

1 | 1 | UNPC-UNK |FOpt |ROHF |6-311G (d) |C12H10 (3) |PCUSER|05-Dec-2003|0||\#P RO HF / 6-311G (D) GUESS=READ OPT SCF (CONVER=5) GEOM=CHECKPOINT $\mid$ mol6-rohf-6 $-311 \mathrm{gd}-3|| 0,3|\mathrm{C}, 1.4315858662,-0.0001409409,0.5684927664| \mathrm{C}, 1.4228339019$ $,-0.0002719776,1.9319622084|\mathrm{H}, 2.3592129503,-0.0003854652,2.460635035| \mathrm{C}$ $, 0.228326933,-0.0002640313,2.6830562729 \mid \mathrm{H}, 0.2767466431,-0.000369268,3$. $7571861232|\mathrm{C},-0.9626775911,-0.0001252481,2.0436896089| \mathrm{H},-1.8851153883$, $-0.0001176973,2.5963641926|C,-1.0168502339,0.0000107826,0.6252966161| C$ $,-2.2827249066,0.0001507182,-0.0166031868 \mid \mathrm{H},-3.1687041613,0.000151938$, $0.5926983025|\mathrm{C},-2.3751400821,0.0002815094,-1.3676893925| \mathrm{H},-3.333822853$ $8,0.0003882179,-1.8541869764 \mid C,-1.2014177926,0.0002749997,-2.142956294$ $|\mathrm{H},-1.2857186178,0.0003777725,-3.2148890036| \mathrm{C}, 0.0466925751,0.000140050$ $2,-1.5715389644|\mathrm{C}, 1.1746790187,0.0001501264,-2.476323397| \mathrm{H}, 1.148255298$ $9,0.0002481675,-3.5487380596 \mid C, 0.1757075819,0.0000056512,-0.1347402893$ $|\mathrm{C}, 2.7755632314,-0.0001707058,-0.1257469074| \mathrm{H}, 2.9107639387,-0.87437536$ $58,-0.7511086823|\mathrm{H}, 3.5680130633,-0.0003096983,0.611567855| \mathrm{H}, 2.91089811$ $58,0.8741457945,-0.7509230337||$ Version=x86-Win32-G98RevA. $11.4 \mid \mathrm{HF}=-460$. $2330422|\mathrm{RMSD}=1.951 \mathrm{e}-006| \mathrm{RMSF}=1.616 \mathrm{e}-004 \mid \mathrm{Dipole}=-0.0937822,0.0000101,-0$ $.0937535|\mathrm{PG}=\mathrm{C} 01 \quad[\mathrm{X}(\mathrm{C} 12 \mathrm{H} 10)]| \mid \varrho$

\section{ROHF/6-311G(d,p)}

1 | 1 | UNPC-UNK | FOpt |ROHF |6-311G (d, p) |C12H10 (3) |PCUSER |08-Dec-2003|0|| \#P ROHF /6-311** OPT GUESS=READ SCF (CONVER=5) GEOM=CHECKPOINT || mol6 || $0,3 \mid \mathrm{C}$ 
$, 1.434745919,-0.000062731,0.559529782 \mid C, 1.4347869972,-0.0013856601,1.9$ $23016585|\mathrm{H}, 2.3752541432,-0.0015521588,2.444589171| \mathrm{C}, 0.2449548394,-0.00$ $16791913,2.6810787125|\mathrm{H}, 0.2996792797,-0.0024050359,3.7550822202| \mathrm{C},-0.9$ $499216215,-0.00063399,2.0494411713 \mid \mathrm{H},-1.8695850109,-0.0005659903,2.606$ $9647437|\mathrm{C},-1.0129294778,0.0003089048,0.6316602906| \mathrm{C},-2.2825472042,0.00$ $11504897,-0.0022486441|\mathrm{H},-3.1642057096,0.0013897873,0.6135342045| \mathrm{C},-2$. $3831021507,0.0016360474,-1.3525966105 \mid \mathrm{H},-3.3451146361,0.0023844921,-1$. $8328346787|\mathrm{C},-1.2149076484,0.0009499892,-2.1354124469| \mathrm{H},-1.3040368428$, $0.0008902698,-3.207125456|\mathrm{C}, 0.0365571599,0.0000631986,-1.5718788856| \mathrm{C}$, $1.1594835097,-0.0018715889,-2.4834748652 \mid \mathrm{H}, 1.1287923373,-0.0025872454$, $-3.5562849839|\mathrm{C}, 0.1749464895,0.0002853907,-0.1360907681| \mathrm{C}, 2.7751562092$ $, 0.0010129043,-0.1429204663 \mid \mathrm{H}, 2.9055085766,-0.8772729916,-0.7654588233$ $|\mathrm{H}, 3.5695567258,0.0061974004,0.5933087941| \mathrm{H}, 2.9008130086,0.8748788918$, $-0.7723983195 \mid$ Version=x86-Win32-G98RevA.11.4 $|\mathrm{HF}=-460.250399| \mathrm{RMSD}=5.50$ $2 \mathrm{e}-006|\mathrm{RMSF}=3.173 \mathrm{e}-005| \mathrm{Dipole}=-0.0771889,0.0001181,-0.0970069 \mid \mathrm{PG}=\mathrm{C} 01 \quad[$ $\mathrm{X}(\mathrm{C} 12 \mathrm{H} 1 \mathrm{O})]|| \mathrm{e}$

\section{ROB3LYP/6-311G(d,p)}

1 | 1 | UNPC-UNK | FOpt |ROB3LYP | 6-311G (d,p) |C12H10 (3) |PCUSER |09-Dec-2003|0|| \#P ROB3LYP / 6-311** OPT GUESS=READ SCF (CONVER=5) GEOM=CHECKPOINT $\mid$ | mO16 $|0,3| C, 1.4369928698,-0.0002682137,0.5568296109 \mid C, 1.4438857211,-0.00044$ $72444,1.9436100382|\mathrm{H}, 2.3976880461,-0.0006096817,2.4602307575| \mathrm{C}, 0.25905$ $99497,-0.0004483423,2.698220281 \mid \mathrm{H}, 0.31006995,-0.0006195364,3.781408296$ $6|\mathrm{C},-0.9541570773,-0.0002217512,2.0574549603| \mathrm{H},-1.8803446051,-0.000200$ $5729,2.6221708453|\mathrm{C},-1.0212586203,0.0000073351,0.6421921442| \mathrm{C},-2.28887$ $93866,0.0002998757,-0.0016258229 \mid \mathrm{H},-3.1819514077,0.0003157663,0.613463$ $0612|\mathrm{C},-2.3856237996,0.0005714476,-1.3767361094| \mathrm{H},-3.3583187875,0.0008$ $204836,-1.8563842056|\mathrm{C},-1.2342407344,0.0005282719,-2.1609335832| \mathrm{H},-1.3$ $183582979,0.0007410211,-3.2414411793 \mid \mathrm{C}, 0.0656166407,0.0002012873,-1.58$ $6385532|\mathrm{C}, 1.1426877507,0.0001217459,-2.4492453563| \mathrm{H}, 1.2181389192,0.000$ $2523039,-3.5278919186|C, 0.1858183226,-0.000039098,-0.1288765207| C, 2.76$ $29712432,-0.000278287,-0.1616076987 \mid \mathrm{H}, 2.8767977879,-0.8794762482,-0.80$ $27300485|\mathrm{H}, 3.5836715911,0.001485629,0.5592148738| \mathrm{H}, 2.8753695266,0.8771$ $286726,-0.8054189502||$ Version=x86-Win32-G98RevA.11.4|HF=-463.2940032|R $\mathrm{MSD}=8.381 \mathrm{e}-006|\mathrm{RMSF}=3.762 \mathrm{e}-005| \mathrm{Dipole}=-0.1024994,-0.0000174,-0.0539807$ $|P G=C 01 \quad[X(\mathrm{C} 12 \mathrm{H} 10)]| \mid @$ 